\title{
Optimizing Adjuvant Therapy for Localized Colon Cancer and Treatment Selection in Advanced Colorectal Cancer
}

\author{
Presented by Axel Grothey, MD, and Alan P. Venook, MD
}

\begin{abstract}
Results from the pivotal IDEA trial, which evaluated 3 versus 6 months of adjuvant oxaliplatin-based chemotherapy, are incorporated into the NCCN Guidelines for Colon Cancer. The guidelines recommend that for patients with low-risk stage III disease, the preferred regimen is CAPEOX for 3 months or FOLFOX for 3 to 6 months. For patients with high-risk stage III disease, the preferred regimen is CAPEOX for 3 to 6 months or FOLFOX for 6 months. In metastatic disease, tumor sidedness should be a consideration when choosing a biologic. For BRAF-mutated disease, several triplets are now recommended options. Importantly, for a subset of patients with metastatic disease, new to the NCCN Guidelines is the incorporation of nivolumab and pembrolizumab as subsequent therapy for those with microsatellite instability-high or mismatch repair-deficient tumors.
\end{abstract}

J Natl Compr Canc Netw 2018;16(5.5):611-615 doi: 10.6004/jnccn.2018.0038

\section{Key Trial Data Inform Colon Cancer Guidelines}

The most significant change to the NCCN Guidelines for Colon Cancer was the incorporation of results from the IDEA trial, according to Axel Grothey, MD, Professor of Oncology and Chair, Division of Hematology and Medical Oncology, Mayo Clinic Cancer Center, who described this study at the NCCN 23rd Annual Conference.

"I believe we have the first important changes in adjuvant chemotherapy since 2004," Dr. Grothey said, referring to the adoption of 6 months of therapy with FOLFOX4 as standard of care, based on results from the MOSAIC trial. ${ }^{1}$

\footnotetext{
Presented by Axel Grothey, MD, Mayo Clinic Cancer Center, Phoenix, Arizona, and Alan P. Venook, MD, University of California, San Francisco, San Francisco, California.

Dr. Grothey has disclosed that he has no financial interests, arrangements, affiliations, or commercial interests with the manufacturers of any products discussed in this article or their competitors. Dr. Venook has disclosed that he has served as a scientific advisor for Bayer HealthCare.

Correspondence: Axel Grothey, MD, Mayo Clinic Cancer Center, 5881 East Mayo Boulevard, Phoenix, AZ 85054.

Email: grothey.axel@mayo.edu; and

Alan P. Venook, MD, UCSF Helen Diller Family Comprehensive Cancer Center, 1450 3rd Street, HD 376, Box 3111, San Francisco, CA 94143-

3111. Email: alan.venook@ucsf.edu
}

Joining Dr. Grothey in describing updates to the NCCN Guidelines was Alan P. Venook, MD, the Madden Family Distinguished Professor of Medical Oncology and Translational Research, and Chief, GI Oncology Clinical Trials Program, University of California, San Francisco. Dr. Venook discussed new recommendations for treating metastatic colon cancer.

\section{IDEA Informs Adjuvant Therapy for Localized Colon Cancer}

The IDEA trial has provided results that will be very helpful for clinicians. ${ }^{2}$ IDEA was a combined analysis of 6 individual studies that determined whether 3 months of adjuvant oxaliplatin-based therapy was as effective as, and less neurotoxic than, 6 months of treatment with the same therapy. The study enrolled 12,834 patients with stage III colon cancer from 20 countries (Figure 1). Of these, 7,763 received FOLFOX (leucovorin/5fluorouracil/oxaliplatin) and 5,071 received CAPEOX (capecitabine/ oxaliplatin) by physician choice. $\mathrm{Pa}$ tients were randomized to 3 or 6 months of adjuvant therapy.

"A high number of patients was needed to make sure, with high confidence, that we are not sacrificing efficacy of therapy for decreased toxicity," Dr. Grothey said. 
Grothey and Venook



Figure 1. IDEA study design $(\mathrm{N}=12,834)$

Abbreviations: DFS, disease-free survival; HR, hazard ratio.

alnvestigator's choice, no randomization.

IDEA was designed as a noninferiority trial in which 3 months of therapy would not sacrifice $>12 \%$ of the benefit of the standard 6 months. In statistical terms, the upper $95 \% \mathrm{CI}$ of the hazard ratio (HR) could not exceed 1.12, to prove noninferiority in 3-year disease-free survival (DFS).

Although the shorter regimen was associated with a dramatic reduction in neurotoxicity, the efficacy end point was not met: 3-year DFS rate was $74.6 \%$ with 3 months of chemotherapy and $75.5 \%$ with 6 months (HR, 1.07; 95\% CI, 1.00-1.15). However, differences were observed according to regimen. For those treated with CAPEOX, 3 months was as good as 6 months, particularly in the lowrisk population. For those treated with FOLFOX, 6 months added extra (although small) benefit in terms of DFS, particularly in the high-risk population, Dr. Grothey reported.

"Importantly, the shorter duration of therapy was associated with a remarkable reduction in neurotoxicity," he added. With FOLFOX, neuropathy (all grades) was observed in $14 \%$ of patients receiving 3 months and in 48\% receiving 6 months of treatment. With CAPEOX, these rates were $15 \%$ and $45 \%$, respectively $(P<.001)$.

\section{Recommendations Based on IDEA}

With FOLFOX, the DFS HR for 3 months' duration was 1.16 (95\% CI, 1.06-1.26), whereas for CAPEOX it was 0.95 (95\% CI, 0.85-1.06), suggesting that the shorter CAPEOX regimen may, in fact, be preferred. Level of recurrence risk also mattered (Table 1). Tumor $(\mathrm{T})$ and nodal $(\mathrm{N})$ stage were grouped together to make a pragmatic choice between low-risk (T1T3,N1) and high-risk (T4 and/or N2). A difference of approximately $20 \%$ at 3 years was shown between these cancers. The $P$ value for interaction by regi-

\begin{tabular}{|lcccl|}
\hline Table 1. Risk-Based, 3-Year Disease-Free \\
Survival of CAPEOX/FOLFOX Combined \\
Risk Group & $\mathbf{3 ~ m o}$ & 6 mo & $\begin{array}{c}\text { HR } \\
(95 \% \mathrm{Cl})\end{array}$ & Conclusion \\
\hline $\begin{array}{l}\text { Low-risk, } \\
\text { regimens } \\
\text { combined }\end{array}$ & 83.1 & 83.3 & $\begin{array}{c}1.01 \\
(0.90-1.12)\end{array}$ & Noninferior \\
\hline $\begin{array}{l}\text { High-risk, } \\
\text { regimens } \\
\text { combined }\end{array}$ & 62.7 & 64.4 & $\begin{array}{c}1.12 \\
(1.03-1.23)\end{array}$ & Inferior \\
\hline $\begin{array}{l}\text { Low-risk } \\
\text { CAPEOX }\end{array}$ & 85.0 & 83.1 & $\begin{array}{c}0.85 \\
(0.71-1.01)\end{array}$ & Noninferior \\
\hline $\begin{array}{l}\text { Low-risk } \\
\text { FOLFOX }\end{array}$ & 81.9 & 83.5 & $\begin{array}{c}1.10 \\
(0.96-1.26)\end{array}$ & Not proven \\
\hline $\begin{array}{l}\text { High-risk } \\
\text { CAPEOX }\end{array}$ & 64.1 & 64.0 & $\begin{array}{c}1.02 \\
(0.89-1.17)\end{array}$ & Not proven \\
\hline $\begin{array}{l}\text { High-risk } \\
\text { FOLFOX }\end{array}$ & 61.5 & 64.7 & $\begin{array}{c}1.20 \\
(1.07-1.35)\end{array}$ & Inferior \\
\hline
\end{tabular}

Low-risk: T1-3,N1; high-risk: T4 and/or N2

Abbreviation: HR, hazard ratio.

men was 0.0061 , but it was not significant by risk group, he added.

The NCCN Guidelines for Colon Cancer, therefore, follow the IDEA recommendations. For patients with low-risk stage III disease (T1-3,N1), the preferred regimen is CAPEOX for 3 months or FOLFOX for 3 to 6 months (category 1 for 6 months' treatment; Figure 2). For patients with high-risk stage III disease (T4,N1-N2 or any $\mathrm{T}$ and N2), the preferred regimen is CAPEOX for 3 to 6 months (category 1 for 6 months) or FOLFOX for 6 months (category 1$)$. Other options include capecitabine $(6$ months) and 5-fluorouracil for 6 months.

"How I interpret IDEA is that the findings are in the eye of the beholder," Dr. Grothey concluded. "A $2 \%$ difference that is statistically inferior might not be of much clinical value to the patient who does not want neurotoxicity. I think we now have a platform for a framework to discuss these data with patients, and that's the strength of IDEA."

\section{Metastatic Colon Cancer: Use of Biomarkers}

As more is learned about the molecular profile of malignant tumors, biomarkers have become "a moving target," Dr. Venook said, "but in colon cancer we have a pretty clear view" of how they should be applied.

The updated NCCN Guidelines spell out what clinicians should look for and expect, especially in 
Localized Colon Cancer and Advanced CRC

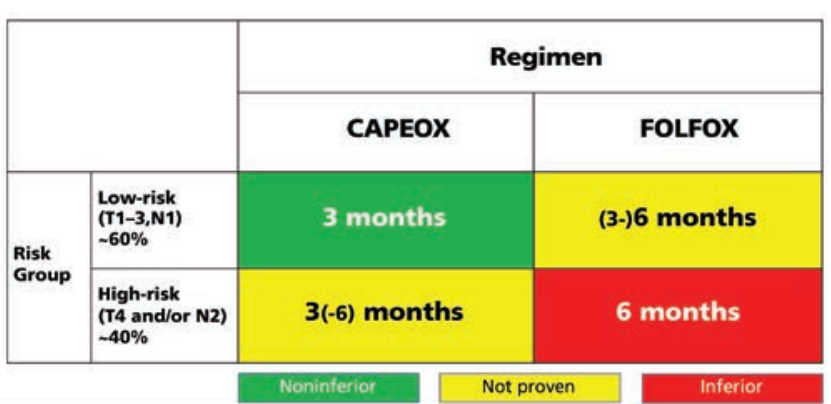

Figure 2. Recommendations from the IDEA study for adjuvant treatment of colon cancer.

advanced disease. Tumors should be evaluated for KRAS, NRAS, and BRAF status, either individually or as part of a next-generation sequencing panel. KRAS and BRAF mutations are mutually exclusive - a fact that can guide the ordering of the tests.

Clinicians should test for the status of microsatellite instability (MSI; also known as mismatch repair [MMR] deficiency). Patients who are MSI-high or MMR-deficient (" 2 ways of looking at the same question") may be candidates for checkpoint inhibitors. These can be identified by immunohistochemistry or gene sequencing.

"We believe that these tests should be run upfront on patients, so you can outline what their treatment choices are," Dr. Venook said.

\section{Chemotherapy Options}

"For choice of chemotherapy-singlets, doublets, triplets, quadruplets-it turns out that 'more' may be better, and this is in the current guidelines," he noted. The guidelines list more than a half dozen regimens and add-ons, the order of which does not reflect preferences (Figure 3). "The options are more or less based on many different parameters."

One good option that may be underused is FOLFOXIRI (fluorouracil/leucovorin/oxaliplatin/irinotecan) with or without bevacizumab. This is based on the results of the TRIBE study that showed FOLOXIRI/bevacizumab produced a 4-month advantage in overall survival (OS) versus FOLFIRI/bevacizumab $(P=.03)$. 3,4 "It doesn't, however, increase your ability to resect metastases, but it really does distinguish outcomes," Dr. Venook observed.

TRIBE also showed the prognostic importance of the BRAF V600E mutation, as patients with that mutation had substantially worse OS. (Other BRAF mutations are not associated with such a poor prognosis.)
In this subgroup, FOLFOXIRI/bevacizumab conferred a 9-month survival benefit versus FOLFIRI (fluorouracil/leucovorin/irinotecan) plus bevacizumab. This regimen, therefore, improves outcomes for patients with poor-risk factors, such as the subset for whom an aggressive approach is important. Because of these findings, Dr. Venook said, "We tend to lean toward FOLFOXIRI as our first-line treatment.”

\section{Cetuximab or Bevacizumab?}

Recent data pertaining to the "sidedness" of the tumor has informed the use of EGFR monoclonal antibodies. Specifically, cetuximab and panitumumab can only be used in KRAS/NRAS wild-type tumors that are located in the left colon.

Two fundamental studies evaluated cetuximab versus bevacizumab as first-line treatment for metastatic RAS wild-type tumors. In FIRE, clear benefit was shown for cetuximab/FOLFIRI over bevacizumab/ FOLFIRI, conferring an 8-month OS benefit..$^{5}$ In Europe, where FIRE-3 was conducted, "Many clinicians accept it as a standard that cetuximab should be used in these patients," Dr. Venook said.

In contrast, CALGB/SWOG 80405, which Dr. Venook led, showed no differences in outcomes between the cetuximab and bevacizumab arms, with FOLFOX or FOLFIRI as backbones. ${ }^{6}$ The discordant result led to some questions, he said, "but as a cooperative group study conducted and interpreted at arm's length from industry, the results of 80405 have not evolved as they did in FIRE-3," he pointed out. Differences in the patient populations, prescribing patterns, and other factors could have contributed to the discrepancy from FIRE-3, he added.

"For all the discussion, in fact, what was pointedly obvious is that the difference in the findings relate to bevacizumab, as opposed to cetuximab," he continued. In patients with all-RAS wild-type disease treated with bevacizumab, median OS was much higher in CALGB/SWOG 80405 than in FIRE-3, whereas survival in the cetuximab arms was similar. "Bevacizumab [arms] did much more poorly in Europe.... We are not sure why. Perhaps some of this is due to the use of bevacizumab at time of progression in the United States," he suggested.

\section{The Issue of Sidedness}

An analysis of outcomes according to tumor sidedness was performed on 1,025 patients from CALGB/ SWOG 80405.7 The analysis showed some differ- 


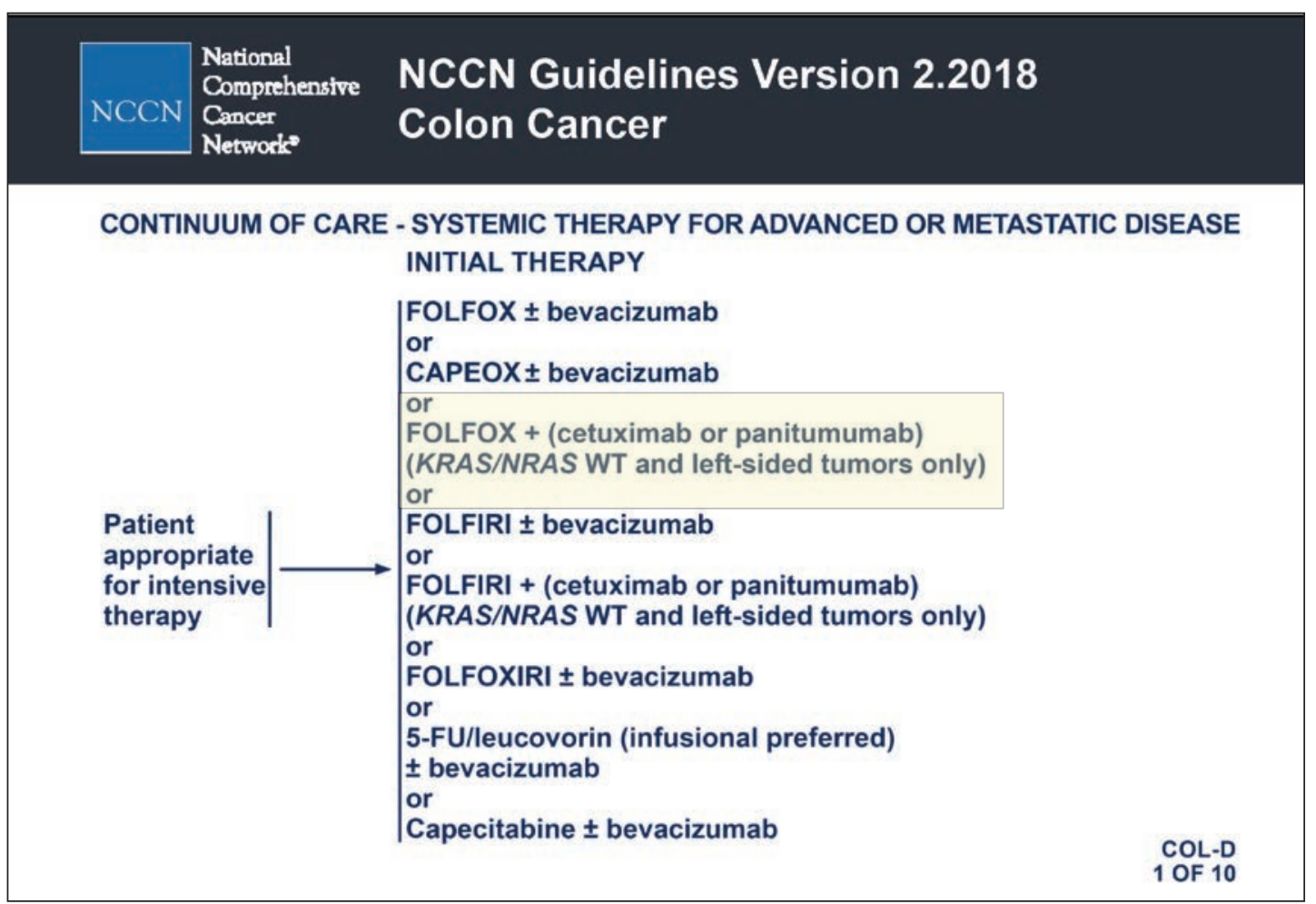

Figure 3. NCCN Guidelines show continuum of care in systemic therapy.

ences in patient characteristics by side, as well as a "dramatically different" difference in treatment outcomes according to the drug they received (Figure 4). Among patients with right-sided tumors, treatment with bevacizumab was associated with longer survival than treatment with cetuximab (24.2 vs 16.7 months). Conversely, among patients with leftsided tumors, treatment with cetuximab was associ-



$$
\begin{array}{ll}
\text { Left/Bev } & \text { Right/Bev } \\
\text { Median }(95 \% \mathrm{Cl}): 31.4(28.3-33.6) & \text { Median }(95 \% \mathrm{Cl}): 24.2(17.9-30.3) \\
\text { Left/Cet } & \text { Right/Cet } \\
\text { Median }(95 \% \mathrm{Cl}): 36.0(32.6-40.3) & \text { Median }(95 \% \mathrm{Cl}): 16.7(13.1-19.4)
\end{array}
$$

Figure 4. Overall survival by sidedness and biologic agent used. Abbreviations: Bev, bevacizumab; Cet, cetuximab. ated with longer OS than bevacizumab (36.0 vs 31.4 months).

The best outcomes were seen in patients with left-sided disease who received cetuximab. They lived fully 15 months longer than those with rightsided disease who received cetuximab (median, 36.0 vs 16.7 months). Similar findings were seen in analyses of other pivotal colon cancer trials. "Clearly, the EGFR antibody cetuximab does not benefit patients with right-sided cancer, independent of their RAS status," Dr. Venook concluded. "We're struggling to figure this out."

Sidedness may not be a factor in the later line use of EGFR antibodies, which have shown some efficacy in this setting, he added, and this is reflected in the guidelines for previously treated patients.

Dr. Venook further pointed out that EGFR antibodies should not be used in patients with potentially resectable synchronous liver and/or lung metastases. This is based on poor outcomes in cetuximab-treated patients in the New EPOC study. ${ }^{8}$ "This is a mystery as to why that would be," Dr. Venook commented. "It's as if the biology of the disease may be different." 


\section{Checkpoint Inhibition in Colon Cancer}

Importantly, for a subset of patients, new to the NCCN Guidelines is the incorporation of nivolum$\mathrm{ab}$ and pembrolizumab as subsequent therapy in patients with MSI-high or MMR-deficient tumors. In a landmark study by Le et $\mathrm{al},{ }^{9} 62 \%$ of patients with colorectal cancer with MMR-deficient tumors experienced response to pembrolizumab. "Clearly these agents have a major impact on this subset of patients," Dr. Venook said.

\section{BRAF-Mutated Tumors}

Also new to the NCCN Guidelines are recommendations for the treatment of BRAF-mutated tumors, an aggressive disease subset with a distinct biology. "A subset of these patients do reasonably well, but most have very rapid disease and a bad result," Dr. Venook noted.

Dr. Venook's group and others have been evaluating the effect of more aggressive therapies in BRAF-mutated disease, using combinations of RAF inhibitors, BRAF inhibitors, and other targeted agents. In a study by Kopetz et al, ${ }^{10}$ progression-free survival was doubled with the triplet of cetuximab/ vemurafenib/irinotecan.

The NCCN Colon Cancer Panel, therefore, recommends combination therapy for tumors with BRAF V600E mutations, including irinotecan/ce- tuximab/vemurafenib and irinotecan/panitumum$\mathrm{ab} /$ vemurafenib.

\section{Looking Ahead}

Drs. Venook and Grothey looked down the road to future revisions in the guidelines. Dr. Venook anticipated a change in regorafenib dosing (which has already happened); and data on the doublet of TAS102 plus bevacizumab will probably lead to changes as well. The results of 2 highly anticipated studies of checkpoint inhibition in microsatellite stable disease (atezolizumab + cobimetinib) and for BRAF-mutated tumors (the triplet of encorafenib/cetuximab/binimetinib) should be available soon.

Dr. Grothey added that "harnessing the idea of immuno-oncology" within the adjuvant setting is also a next step. "We know that MSI-high and MMRdeficient tumors respond well in the palliative setting, with high response rates and long durations of response, so why wouldn't checkpoint inhibitors work in the adjuvant setting too?" he commented. The ATOMIC study has been initiated to evaluate atezolizumab in the adjuvant setting for MMR-deficient colon cancers. "This is a very interesting study and it can hopefully set a new standard of care," he said.

\section{References}

1. Andre $\mathrm{T}$, Boni $\mathrm{C}$, Mounedji-Boudiaf L, et al. Oxaliplatin, fluorouracil, and leucovorin as adjuvant treatment for colon cancer. N Engl J Med 2004;350:2343-2351.

2. Shi Q, Sobrero AF, Shields AF, et al. Prospective pooled analysis of six phase III trials investigating duration of adjuvant oxaliplatin-based therapy ( 3 vs 6 months) for patients with stage III colon cancer: the IDEA (International Duration Evaluation of Adjuvant Chemotherapy) Collaboration [abstract]. J Clin Oncol 2017;35(Suppl):Abstract LBA1.

3. Loupakis F, Cremolini C, Masi G, et al. Initial therapy with FOLFOXIRI and bevacizumab for metastatic colorectal cancer. $N$ Engl J Med 2014;371:1609-1618.

4. Cremolini C, Loupakis F, Antoniotti C, et al. FOLFOXIRI plus bevacizumab versus FOLFIRI plus bevacizumab as first-line treatment of patients with metastatic colorectal cancer: updated overall survival and molecular subgroup analyses of the open-label, phase 3 TRIBE study. Lancet Oncol 2015;16:1306-1315.

5. Stintzing S, Modest DP, Rossius L, et al. FOLFIRI plus cetuximab versus FOLFIRI plus bevacizumab for metastatic colorectal cancer (FIRE-3): a post-hoc analysis of tumour dynamics in the final RAS wild-type subgroup

of this randomized open-label phase 3 trial. Lancet Oncol 2016;17:14261434.

6. Venook AP, Niedzwiecki D, Lenz HJ, et al. Effect of first-line chemotherapy combined with cetuximab or bevacizumab on overall survival in patients with KRAS wild-type advanced or metastatic colorectal cancer: a randomized clinical trial. JAMA 2017;317:2392-2401.

7. Venook AP, Niedzwiecki D, Innocenti F, et al. Impact of primary $\left(1^{\circ}\right)$ tumor location on overall survival (OS) and progression-free survival (PFS) in patients (pts) with metastatic colorectal cancer (mCRC): analysis of CALGB/SWOG 80405 (Alliance) [abstract]. J Clin Oncol 2016;34(Suppl):Abstract 3504.

8. Primrose J, Falk S, Finch-Jones M, et al. Systemic chemotherapy with or without cetuximab in patients with resectable colorectal liver metastasis: the New EPOC randomised controlled trial. Lancet Oncol 2014;15:601611.

9. Le DT, Uram JN, Wang H, et al. PD-1 blockade in tumors with mismatchrepair deficiency. N Engl J Med 2015;372:2509-2520.

10. Kopetz S, McDonough SL, Morris VK, et al. Randomized trial of irinotecan and cetuximab with or without vemurafenib in BRAF-mutant metastatic colorectal cancer (SWOG 1406) [abstract]. J Clin Oncol 2017;35(Suppl):Abstract 520. 